\title{
Rare stimuli or rare changes: what really matters for the brain?
}

\author{
Veronica Mazza, ${ }^{\text {,CA }}$ Massimo Turatto ${ }^{3}$ and Michela Sarlo ${ }^{2}$
}

\begin{abstract}
'Departments of Developmental Psychology; ${ }^{2}$ General Psychology, University of Padova, Padova, Italy; ${ }^{3}$ Department of Cognitive Science and Education, University of Trento, Rovereto, Italy

${ }^{\mathrm{CA} C o r r e s p o n d i n g ~ A u t h o r: ~ v e r o n i c a . m a z z a @ u n i p d . i t ~}$
\end{abstract}

Received I8 March 2005; accepted 28 April 2005

Previous research has demonstrated that the electric brain response to changes consists of a negative deflection with a latency of 200 ms post-stimulus. Two different hypotheses, namely the mismatch and the rareness accounts, have been invoked to explain this electrocortical response. In the present study, this negative component emerged only for visual changes caused by the presentation of rare stimuli, documenting a precise brain response to rareness, and not to change per se. Crucially, no specific electrophysiological marker was evident when the change was rare, but consisted of stimuli frequently seen. Hence, we suggest that changes are preferentially processed by the brain only when they involve the occurrence of new and rare stimuli. NeuroReport 16:1061-1064 (c) 2005 Lippincott Williams \& Wilkins.

Key words: Change processing; Event-related potentials; N2; Rareness; Relevant visual changes; Visual mismatch negativity

\section{INTRODUCTION}

In the past decade, the study of change processing has evoked new interest, because of the so-called change blindness phenomenon [1,2]. Numerous investigations on change blindness have shown striking limitations in our conscious perception of changes to visual scenes [3-5]. However, change processing was already an important issue in psychophysiological research, where several eventrelated potential (ERP) studies have consistently shown the occurrence of a negative deflection in the N2 latency range (150-300 ms), elicited by the change onset [6]. Specifically, the mismatch negativity (MMN) is most frequently indicated as the brain's electrical response to stimulus change.

The MMN is commonly evoked by rare or novel stimuli embedded in a series of frequent stimuli. Initially examined within the auditory modality [7], the existence of a visual MMN (vMMN) has been recently investigated [8-15]. Two different interpretations have been proposed about the mechanisms that would trigger the vMMN. According to the memory trace hypothesis, the vMMN would result from a comparison between a memory trace of a recent stimulation (frequent stimuli), and the representation of the current stimulation (rare stimuli) $[8,10,12,13,15]$. By contrast, the rareness account states that frequent presentations of a stimulus induce saturation of the neural populations that respond to that stimulus [11]. Hence, the vMMN would occur because neurons can maintain their responsiveness to stimuli when they are presented with infrequent rates.

Because this N2-like component has often been associated with stimulus change processing, it might be considered as an ideal candidate to examine electrophysiological correlates of rare change perception. However, in most of the previous studies, the infrequent change was task-irrelevant (e.g. [16,17]). Therefore, unequivocal and direct links between N2-like components and explicit change perception cannot be made yet. The aim of the present study was to examine the electrophysiological correlates of task-relevant visual changes, with particular interest to N2-like components. To this purpose, participants were explicitly instructed to search for a change, and to report its presence or absence at the end of each trial. In assessing ERP correlates of relevant change perception, we also manipulated the probability of change occurrence. In the first condition, the change was rare (20\% of total trials), and could involve the presentation of either a frequent or a rare stimulus. That is, two types of change could occur, the first consisting of the presentation of an unpredictable rare stimulus (e.g. a red square) after a series of frequent stimuli (e.g. green circles), whereas the second consisted of the successive return to the frequent stimulus (e.g. a green circle). This manipulation was motivated by the fact that a change, even when rare, does not necessarily involve the presentation of a rare stimulus. Indeed, any stimulus that is different from the previous one, regardless of its presentation rate, can be considered as a change. On the other hand, a change itself does not have to be a rare event, as it can simply be defined as the alteration of an object over time [2]. Therefore, in the second condition, the change was a frequent event, involving the presentation of equiprobable and unpredictable stimuli. In this regard, some evidence exists that, for both auditory and visual modalities, a deviance negativity can be elicited even by equiprobable stimuli $[18,10]$.

Change perception relies essentially on comparison processes between the current stimulus and the mnestic 
representation of the previous one. According to the memory trace hypothesis, we could predict a deviance negativity on each change trial. Alternatively, the emergence of a negative component only on trials in which rare stimuli are presented would be better explained by the rareness account, and not necessarily linked to change perception as such.

\section{METHODS}

Eleven healthy students (seven women; nine right-handed; aged 20-29 years, mean age 23) at the University of Padua participated in the experiment, after providing informed consent. All reported normal or corrected visual acuity and normal colour vision. The study was approved by the local ethical committee.

Participants sat in a dimly lit, electrically shielded room, at a viewing distance of $1 \mathrm{~m}$ from a 19-inch colour monitor $(640 \times 480,75 \mathrm{~Hz})$. A central white cross $\left(0.6^{\circ}\right)$ at the centre of the screen served as a fixation point, and was presented throughout each block of trials. A red square or an isoluminant green circle (each approximately covering $2.9^{\circ}$ ) appeared to the left or right of the fixation point, at a distance of $4.7^{\circ}$. In the rare-change condition, squares and circles were presented according to a 1:9 rate, in a pseudorandom sequence, with the restriction that two rare stimuli would never be presented successively. The type of stimuli which served as rare $(10 \%)$ and frequent stimulus (90\%) were counterbalanced across participants. Change occurrence was set at $20 \%$ of the total trials, half of which consisted of the unpredictable presentation of rare stimuli on trials $n$, whereas the remainder consisted of the presentation of frequent stimuli on trials $n+1$. In the equiprobable-change condition, squares and circles were presented in a random sequence, with equal probability. In both conditions, stimulus location (left or right) was kept constant throughout each block. The order of the rarechange and equiprobable-change condition was counterbalanced across participants. The experimental session consisted of four blocks, with 300 trials per block. On each trial, the stimulus was presented simultaneously with the fixation cross for $500 \mathrm{~ms}$. Then, $300 \mathrm{~ms}$ after stimulus offset, the central cross turned blue, prompting participants to report whether the current stimulus had changed from the previous one. Participants had $1700 \mathrm{~ms}$ to respond, by pressing a left key to indicate change presence and a right key to indicate the absence of a change. A training block of 10 trials was performed before the start of each experimental block. Participants were instructed to maintain their gaze at fixation, and to respond only when prompted. They were also invited to minimize eye blinks and to avoid any unnecessary movement.

Electroencephalogram (EEG) data were recorded from 60 tin electrodes mounted in an elastic cap (according to the 10-20 system) and referred to the tip of the nose. A vertical electrooculogram (EOG) was recorded bipolarly with two electrodes below and above the left eye, and a horizontal EOG with electrodes on the external canthus of each eye. Impedance was kept below $5 \mathrm{k} \Omega$ for all electrodes, amplifier bandpass was $0.1-40 \mathrm{~Hz}$ and digitization rate was $500 \mathrm{~Hz}$. EEG and EOG were epoched offline from -100 to $800 \mathrm{~ms}$ relative to stimulus onset. Epochs with eyeblinks, eye movements or any other artefacts (EEG exceeding $\pm 70 \mu \mathrm{V}$ ) were excluded. Averages were computed separately for each stimulus location (left, right) and stimulus type (for the rare-change condition: no change, rare stimulus and frequent stimulus; for the equiprobable-change condition: no change, equiprobable stimulus), relative to a $100 \mathrm{~ms}$ prestimulus baseline. After filtering the averages with a low-pass filter $(20 \mathrm{~Hz}, 24 \mathrm{~dB} / \mathrm{oct})$, difference waveforms were calculated by subtracting ERPs elicited by no-change trials from those elicited by change trials for the same condition, separately for stimulus location. To reduce the electrode number, three regions of eight electrode pairs were chosen: lateral anterior (AF3, AF4, F7, F5, F3, F1, F2, F4, F6, F8, FC5, FC3, FC1, FC2, FC4, FC6), lateral central (T7, C5, C3, C1, C2, C4, C6, T8, TP7, CP5, CP3, CP1, CP2, CP4, CP6, TP8) and lateral posterior (P7, P5, P3, P1, P2, P4, P6, P8, PO7, $\mathrm{PO} 5, \mathrm{PO} 3, \mathrm{PO} 4, \mathrm{PO} 6, \mathrm{PO} 8, \mathrm{O} 1, \mathrm{O} 2)$ electrodes.

Mean difference amplitudes for each region, separately for symmetrical left and right electrodes, were submitted to repeated-measures ANOVAs, with the factors type (rare stimulus, frequent stimulus, equiprobable stimulus), stimulus location (left, right), region (anterior, central and posterior) and hemisphere (left, right). ANOVAs were conducted for the following time windows: $200-300 \mathrm{~ms}$ (N2 range) and 300-600 ms (P3) post-stimulus onset. When appropriate, Greenhouse-Geisser corrections for sphericity violations were applied and corrected $p$-values are reported. Significant single effects were further analysed by means of pairwise comparisons ( $t$-tests). To minimize type II errors while controlling type I errors, we adjusted the $\alpha$ level for the number of comparisons, for both behavioural and ERP data, following the false discovery rate procedure [19]. Only significant effects (with corrected $p$-values) are reported.

\section{RESULTS}

In the rare-change condition, participants responded correctly on $97 \%$ of the change trials that involved a frequent stimulus and on $100 \%$ of the trials that involved a rare stimulus. Although very small, this difference was significant, $t(10)=3.35, p<0.008$. In the equiprobable-change condition, participants were less accurate (90\%) than in previous conditions, with both $t \mathrm{~s}(10)>3.32$, both $p \mathrm{~s}<0.009$. False alarm rates for the rare-change and equiprobablechange conditions were $2 \%$ and $9 \%$, respectively.

Figure 1a shows ERP difference waveforms for rare, frequent and equiprobable stimuli. Two main differences emerged. Rare stimuli elicited a prominent negativity, starting at lateral posterior regions at about $200 \mathrm{~ms}$ and then spreading to more anterior sites (Fig. 1b). This modulation was followed by a broadly distributed positivity, starting at about $300 \mathrm{~ms}$. No such differences were present for frequent or equiprobable stimuli. From the ANOVA, in the $\mathrm{N} 2$ range (200-300 ms), the effect of type was significant, $\mathrm{F}(2,20)=8.887, p<0.008$, as were the type $\times$ region interaction, $\mathrm{F}(4,40)=17.685, p<0.0002$ and the type $\times$ hemisphere interaction, $F(2,20)=3.723, p<0.043$. The first interaction was further analysed by means of three ANOVAs, separately for each region. The effect of type was significant at central and posterior regions only, both Fs $(2,20)>9.237$, both $p s<0.008$. At these regions, the rare stimuli elicited a more pronounced N2 than frequent and equiprobable stimuli, with all $t_{\mathrm{s}}(10)>2.942$, all $p \mathrm{~s}<0.016$. By contrast, no significant difference emerged between frequent and equiprobable stimuli, the lowest $p=0.77$. In addition, neither of these conditions differed significantly 
(a)

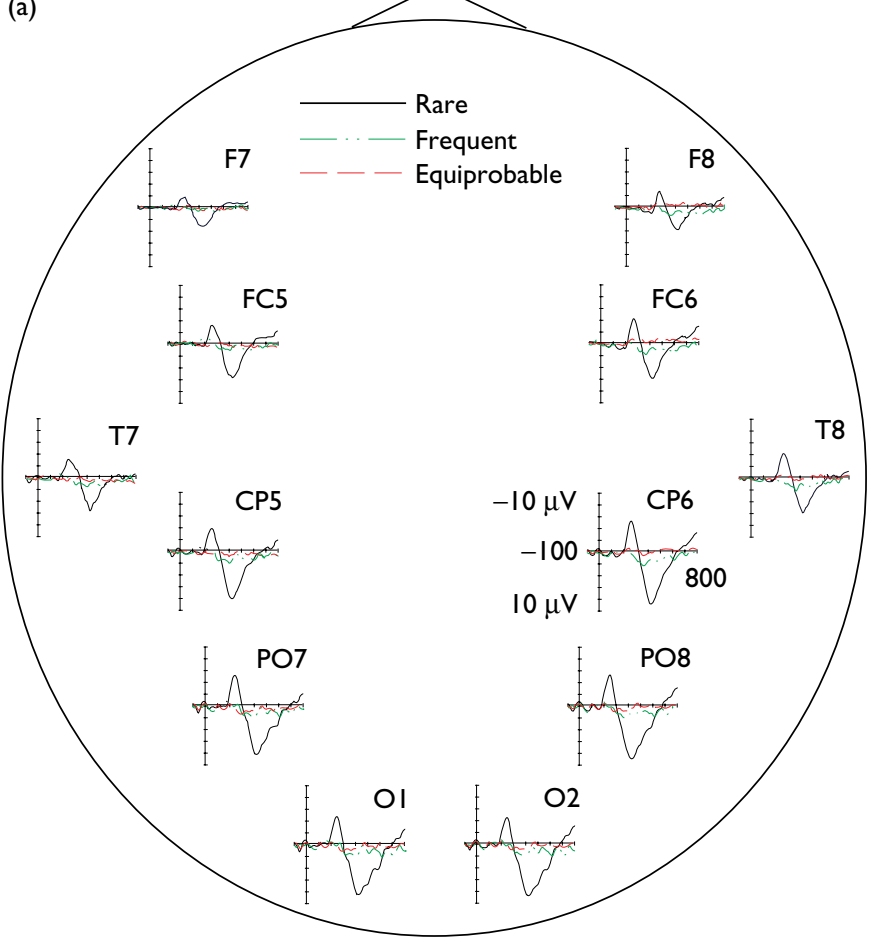

(b)
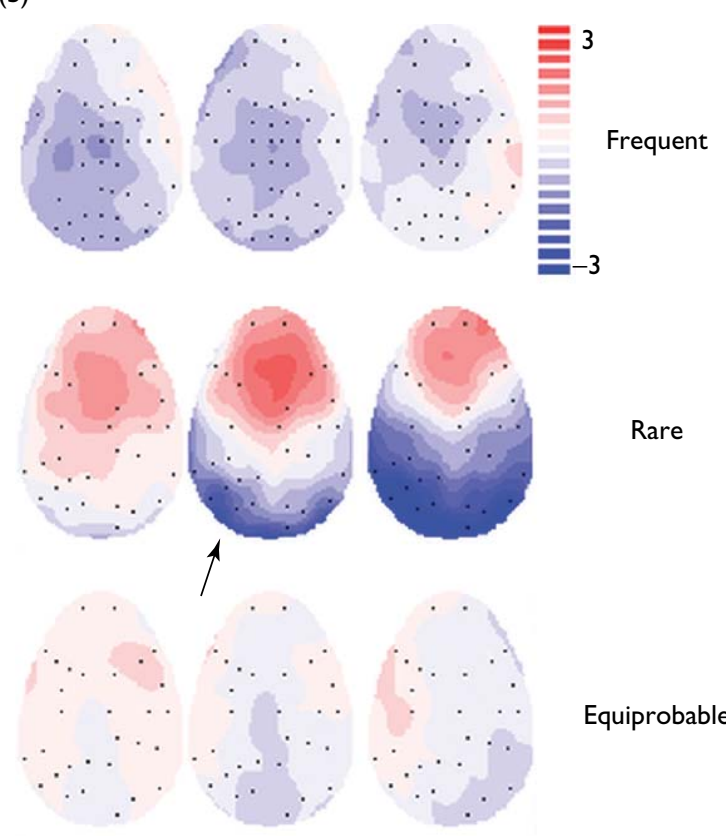

Rare

$180 \mathrm{~ms}$

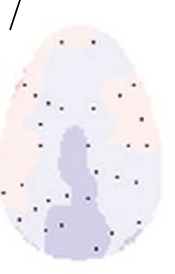

$200 \mathrm{~ms}$
$220 \mathrm{~ms}$

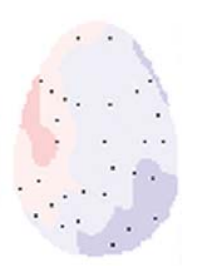

Fig. I. (a) Grand-averaged event-related potential difference waveforms for frequent (dash-and-dot lines), rare (solid lines) and equiprobable (dashed lines) stimuli. Two representative pairs of electrodes for each region are shown. (b) Topographical scalp distribution of the difference waves from 180 to $220 \mathrm{~ms}$ from stimulus onset. The negative component elicited by rare stimuli started at the most posterior sites, as pointed by the arrow.

from zero, the lowest $p=0.56$, thus indicating that change trials with frequent and equiprobable stimuli did not differ from no-change trials. The same pattern of results, with a more pronounced N2 for the change involving rare stimuli than the other two stimuli, was also found in the two ANOVAs aimed to clarify the type $\times$ hemisphere interaction, separately for left and right hemisphere, all $p s<0.027$ (Fig. 1a). That is, no hemispheric asymmetries were found.

The ANOVA in the P3 range $(300-600 \mathrm{~ms})$ revealed significant effects of type, $\mathrm{F}(2,20)=26.69, p<0.0001$, type $\times$ region, $\mathrm{F}(4,40)=26.851, p<0.0001$, and type $\times$ hemisphere, $\mathrm{F}(2,20)=5.589, p<0.013$. As above, three separate ANOVAs were used for exploring the first significant interaction. Unlike the previous analyses, the effect of type was significant at all regions, with all $\mathrm{Fs}(2,20)>10.381$, all $p s<0.002$. Again, rare stimuli elicited a more pronounced P3 than frequent and equiprobable stimuli at central and posterior regions, with all $t_{\mathrm{s}}(10)>4.164$, all $p \mathrm{~s}<0.003$. However, at the anterior region, rare stimuli elicited a more pronounced positivity than only equiprobable stimuli, $t(10)=5.201, p<0.0005$. In addition, at variance with the analysis in the N2 range, the P3 for frequent stimuli was significantly greater than zero at posterior regions, $t(10)=2.899, p<0.017$. As for the significant type $\times$ hemisphere interaction in the N2 analysis, a more pronounced P3 was found for rare stimuli than for the other two types at both hemispheres (all $p \mathrm{~s}<0.003$ ), with no evidence of leftright asymmetries.

\section{DISCUSSION}

The present study aimed to examine ERP correlates of relevant changes by using an explicit change detection task.
The crucial result was that in the rare-change condition, rare stimuli elicited a prominent negativity, with a latency of about $200 \mathrm{~ms}$, starting at posterior regions and then spreading towards central regions (Fig. 1b), in line with previous results on the vMMN [11-13]. Because the occurrence of a rare stimulus after a frequent stimulus, and the successive return to the frequent stimulus, could be conceived as a visual change, one possibility would have been to find a change-related negativity for either type of stimuli. The lack of such a result, however, indicates that this negative component cannot be considered the correlate of change perception as such. Indeed, our results showed that this negative component correlates only with the presentation of rare and new stimuli, which, however, necessarily involve a change. A second ERP modulation was found in the P3 latency range, with a more pronounced positivity for rare stimuli than for the other two types of stimuli (Fig. 1a). However, as frequent stimuli also elicited a P3 component at posterior regions, it is rather difficult to consider this positivity as a correlate of rare stimuli occurrence. In this case, the rareness interpretation appears to be less convincing, and requires further research to be fully understood.

Before accepting the interpretation of the negative component as a specific brain response to rare stimuli, alternative accounts need to be considered.

Contrary to previous studies on visual change processing (e.g. [11-13]), we used a more direct approach, making rare changes relevant for the task. Thus, one may wonder whether the negative deflection for rare stimuli could be better explained in terms of attentional processing of taskrelevant features $[20,21]$. However, in our study, all stimuli were task-relevant, that is, all stimuli, regardless of change 
occurrence, were to be processed attentively and required an overt response. Hence, the negative component elicited by rare stimuli cannot be explained totally in terms of attentional processing of task-relevant stimuli. A related issue concerns the behavioural findings, which showed higher accuracy for rare stimuli than for frequent and equiprobable stimuli. Thus, task difficulty might have accounted for the emergence of the negative component, with greater accuracy eliciting greater negativity [22,23]. However, had task difficulty played a relevant role for the occurrence of the negative deflection, we should have found a similar difference also between frequent and equiprobable stimuli, wherein an even greater difference in accuracy percentage (97\% vs. $90 \%$ ) was measured, which clearly was not the case.

A second issue refers to the difference in predictability of stimuli rather than to the rareness of their presentation. While participants could not predict the occurrence of rare stimuli, it is possible, if not likely, that they could anticipate that a frequent stimulus would immediately follow the rare one. However, the comparison between rare and equiprobable conditions, where also highly unpredictable stimuli were used, showed a negative component for rare stimuli only, suggesting that predictability is not crucial for the occurrence of this component. This was in turn substantiated by the lack of significant differences between (predictable) frequent stimuli and (unpredictable) equiprobable stimuli.

Finally, a recent ERP study on change blindness found that successful change detection of lateralized stimuli is correlated to a negative deflection, contralateral to the change location [24]. Although the present findings might seem at odds, in that no evidence of negative deflection was found for change trials as such, it may be noted that, in the Eimer and Mazza study, change detection probably required continuous shifts of attention towards the location of change. By contrast, here the change location was kept constant throughout each block, thus requiring a single shift of attention to the location of the change at the beginning of each block of trials.

\section{CONCLUSION}

Our study points to the presence of a negative component, with a latency of $200 \mathrm{~ms}$ at posterior sites, as the electrical counterpart of rare change processing, only when this is accompanied by the presentation of rare stimuli. This finding is in agreement with the idea that, at least in simple experimental situations as the one used here, the visual system relies on mechanisms that compute the rareness of events, rather than comparing the current stimulus with a mnestic trace of the previous one.

\section{REFERENCES}

1. Simons DJ, Levin DT. Change blindness. Trends Cogn Sci 1997; 1: 261-267.

2. Rensink RA. Change detection. Annu Rev Psychol 2002; 53:245-277.

3. Rensink RA, O'Regan JK, Clark JJ. To see or not to see: the need for attention to perceive changes in scenes. Psychol Sci 1997; 8: 368-373.

4. Turatto M, Angrilli A, Mazza V, Umiltà C, Driver J. Looking without seeing the background change: electrophysiological correlates of change detection versus change blindness. Cognition 2002; 84:B1-B10.

5. Turatto M, Sandrini M, Miniussi C. The role of the right dorsolateral prefrontal cortex in visual change awareness. Neuroreport 2004; 15: 2549-2552.

6. Pazo-Alvarez P, Cadaveira F, Amenedo E. MMN in the visual modality: a review. Biol Psychol 2003; 63:199-236.

7. Näätänen R, Gaillard AWK, Mäntysalo S. Early selective-attention effect on evoked potential reinterpreted. Acta Psychol (Amst) 1978; 42:313-329.

8. Czigler I, Balázs L, Winkler I. Memory-based detection of task-irrelevant visual changes. Psychophysiology 2002; 39:869-873.

9. Czigler I, Balázs L, Pató L. Visual change detection: event-related potentials are dependent on stimulus location in humans. Neurosci Lett 2004; 364:149-153.

10. Fu S, Fan S, Chen L. Event-related potentials reveal involuntary processing of orientation changes in the visual modality. Psychophysiology 2003; 40:770-775.

11. Kenemans JL, Grent-'t Jong T, Verbaten MN. Detection of visual change: mismatch or rareness? Neuroreport 2003; 14:1239-1242.

12. Stagg C, Hindley $P$, Tales A, Butler S. Visual mismatch negativity: the detection of stimulus change. Neuroreport 2004; 15:659-663.

13. Tales A, Newton P, Troscianko T, Butler S. Mismatch negativity in the visual modality. Neuroreport 1999; 10:1-5.

14. Woods DL, Alho K, Algazi A. Intermodal selective attention. I. Effects on event-related potentials to lateralized auditory and visual stimuli. Electroencephalogr Clin Neurophysiol 1992; 82:341-355.

15. Astikainen P, Ruusuvirta T, Wikgren J, Korhonen T. The human brain processes visual changes that are not cued by attended auditory stimulation. Neurosci Lett 2004; 368:231-234.

16. Czigler I, Csibra G. Event-related potentials in a visual discrimination task: negative waves related to detection and attention. Psychophysiology 1990; 27:669-676.

17. Berti S, Schroger E. A comparison of auditory and visual distraction effects: behavioral and event-related indices. Brain Res Cogn Brain Res 2001; 10:265-273.

18. Sams M, Alho K, Näätänen R. Short-term habituation and dishabituation of the mismatch negativity of the ERP. Psychophysiology 1984; 21: 434-441.

19. Benjamini Y, Hochberg Y. Controlling the False Discovery Rate: A practical and powerful approach to multiple testing. J R Statist Soc B 1995; 57:289-300.

20. Csibra G, Czigler I. Event-related potentials to irrelevant deviant motion of visual shapes. Int J Psychophysiol 1991; 11:155-159.

21. Hillyard SA, Anllo-Vento L. Event-related brain potentials in the study of visual selective attention. Proc Natl Acad Sci 1998; 95:781-787.

22. Czigler I, Balázs L. Event-related potentials and audiovisual stimuli: multimodal interactions. Neuroreport 2001; 12:223-226.

23. Smid HGOM, Jakob A, Heinze HJ. An event-related brain potential study of visual selective attention to conjunctions of color and shape. Psychophysiology 1999; 36:264-279.

24. Eimer M, Mazza V. Electrophysiological correlates of change detection. Psychophysiology (in press). 